\title{
A Mathematical Model for the Dynamics of a Fish Algae Consumption Model with Impulsive Control Strategy
}

\author{
Jin Yang ${ }^{1}$ and Min Zhao ${ }^{2}$ \\ ${ }^{1}$ School of Mathematics and Information Science, Wenzhou University, Zhejiang, Wenzhou 325035, China \\ ${ }^{2}$ School of Life and Environmental Science, Wenzhou University, Zhejiang, Wenzhou 325035, China
}

Correspondence should be addressed to Min Zhao, zmcn@tom.com

Received 21 October 2011; Accepted 7 February 2012

Academic Editor: Leevan Ling

Copyright (c) 2012 J. Yang and M. Zhao. This is an open access article distributed under the Creative Commons Attribution License, which permits unrestricted use, distribution, and reproduction in any medium, provided the original work is properly cited.

\begin{abstract}
A dynamic mathematical model of fish algae consumption with an impulsive control strategy is proposed and analyzed in detail. It is shown that the system has a globally asymptotically stable algae-eradication periodic solution which can be obtained using the Floquet theory of impulsive differential equations and small-amplitude perturbation techniques. The conditions for the permanence of the system can also be determined. Numerical results for impulsive perturbations show the rich dynamic behavior of the system. All these results may be useful in controlling eutrophication.
\end{abstract}

\section{Introduction}

One of the most common ecological and environmental problems of inland water bodies is eutrophication, which diminishes water quality by spurring excessive growth of algae and increasing amounts of suspended organic material [1-3]. Because of climate change and discharges of industrial wastewater into rivers, the self-purification capacity of the water body decreases. This provides favorable conditions for algae growth, leading to repeated outbreaks of abnormal proliferations of algae blooms in tributaries; recently, this phenomenon has been increasing [4]. Therefore, research on how to control the amount of algae in the environment is of great theoretical importance and practical significance.

There are many ways to control algae populations. The most widely used method is chemical control; this is not discussed in detail here because it has many negative impacts. More recently, another important method, called biological control, has been introduced. Biological control is the practice of using natural enemies (fish) to suppress algae, as has 
already been done for pest control [5-8]. These natural enemies can prevent algae populations from increasing to levels that cause eutrophication.

Mathematical models of ecological population dynamics have not only to account for growth and interactions, but also to improve the understanding of the functioning of food chains and webs and their dependence on some environmental conditions [9-12]. Impulsive differential equations have been widely used to study the mathematical properties of impulsive predator-prey models or three-species food-chain models [13-17]. Moreover, the theory of impulsive differential equations is being recognized, not only to be richer than the corresponding theory of differential equations without impulses, but also to represent a more natural framework for the mathematical modeling of real-world phenomena [18-20].

Recently, because of eutrophication, the nuisance algal (cyanobacteria and green algae) blooms frequently come forth in the Zeya reservoir which is located in Wenzhou. The algal blooms can cause clogging and blocking of the filtration system and result in millions of people without drinking water. In order to apply the biological principle to control algal blooms in the Zeya reservoir, we carry out good biological control agents for the algal blooms. Firstly, we bring up two species of filter-feeding fish, silver carp (Hypophthalmichthys molitrix) and bighead carp (Aristichthys nobilis), which are thought to be suited to control algal biomass directly in freshwater reservoir, especially cyanobacteria and green algae. Secondly, the decision maker must give cost-benefit and maintenance considerations; the fish should be harvested at an appropriate time. Finally, in order to prevent the rapid growth of the cyanobacteria and green algae, we must release a certain amount of filter-feeding fish at a fixed time. These processes are subject to short-term perturbations. Consequently, it is obvious to assume that these perturbations act instantaneously in the form of impulses. Hence, a fish algae consumption model can be described by the following differential equations:

$$
\begin{aligned}
& \frac{d x(t)}{d t}=r_{1} x(t)\left(\frac{G_{0}-x(t)}{G_{1}-x(t)}\right)-c_{1} x^{2}(t)-\frac{u_{1} x(t) z(t)}{b_{1}+x(t)} \\
& \frac{d y(t)}{d t}=r_{2} y(t)\left(\frac{1-\left(y(t) / y_{m}\right)}{1-\left(y(t) / y_{n}\right)}\right)-c_{2} y^{2}(t)-\frac{u_{1} a y(t) z(t)}{x(t)+a y(t)+b_{2}}, \quad t \neq n T, \\
& \frac{d z(t)}{d t}=-m z(t)+\frac{u_{2} a y(t) z(t)}{x(t)+a y(t)+b_{2}}+\frac{u_{3} x(t) z(t)}{b_{1}+x(t)} \\
& \Delta x(t)=0, \\
& \Delta y(t)=0, \quad t=n T \\
& \Delta z(t)=-\delta z(t)+p
\end{aligned}
$$

where $x(t), y(t), z(t)$ are the densities of the cyanobacteria population, green algae population, and fish population at time $t, \Delta x(t)=x\left(t^{+}\right)-x(t) ; \Delta y(t)=y\left(t^{+}\right)-y(t)$, and $\Delta z(t)=z\left(t^{+}\right)-z(t) ; r_{1}, r_{2}$ is a growth parameter which is related to the biological characteristics of populations and the rationalization of environmental resources; $r_{1} G_{0}(0 \leq$ $\left.G_{0} \leq G_{1}\right)$ is the carrying capacity of the cyanobacteria population $x ; G_{1}$ is the limiting value of available resources; $y_{m}\left(0 \leq\left(y_{m} / y_{n}\right) \leq 1\right)$ is the maximum density of green algae population $y$ (i.e., the environmental carrying capacity); $y_{n}$ is a nutritional parameter which is related to the resource conditions of the environment; $u_{1}$ is the cropping rate of fish; $u_{2}$ and $u_{3}$ are the 
average growth rate of fish that depends on the cyanobacteria and green algae for food; $a$ is the capture rate (as a proportion); $m$ is the average mortality rate for fish; $b_{1}$ and $b_{2}$ are the half-saturation constants; $c_{1}$ and $c_{2}$ are the intraspecific competition rates of the cyanobacteria and green algae; $T$ is the period of the impulsive effect; $N$ is the set of all nonnegative integers; $\delta(\delta \in[0,1])$ is the proportion of fish harvested at fixed times $t=n T$; and $p>0$ is the number of fish released at times $t=n T$.

The rest of this paper is arranged as follows: in the next section, some useful notations and basic properties of a system with impulsive effect are given, and then the main theorems and their proofs are developed using the Floquet theory. In Section 3, numerical results will be presented which exhibit the rich dynamic behaviors of system (1.1), and these results will be briefly discussed. Conclusions are given in the last section.

\section{Preliminaries and Mathematical Analysis}

Let $R_{+}=[0, \infty), R_{3}^{+}=\left\{X \in R^{3} \mid X>0\right\}$. Denote by $f=\left(f_{1}, f_{2}, f_{3}\right)$ the mapping defined by the right-hand sides of the first, second, and third equations of system (1.1). Let $V: R_{+} \times R_{+}^{3} \rightarrow R_{+}$; then $V$ is said to belong to class $V_{0}$ if

(1) $V$ is continuous in $(n T,(n+1) T] \times R_{+}^{3}$, and for each $X \in R_{+}^{3}, n \in N$, $\lim _{(t, y) \rightarrow\left(n T^{+}, X\right)} V(t, y)=V\left(n T^{+}, X\right)$ exists.

(2) $V$ is locally Lipschitzian in $X$.

Definition 2.1 (see [18]). Let $V \in V_{0}$; then for $(t, x) \in(n T,(n+1) T] \times R_{+}^{3}$, the upper right derivative of $V(t)$ with respect to the impulsive differential system (1.1) is defined as

$$
D^{+} V(t, X)=\lim _{h \rightarrow 0^{+}} \sup \frac{1}{h}[V(t+h, X+h f(t, X))-V(t, X)] .
$$

Remark 2.2. (1) The solution of system (1.1) is a piecewise continuous function with $X: R_{+} \rightarrow$ $R_{+}^{3} ; X(t)$ is continuous on $(n T,(n+1) T]$, and $X\left(n T^{+}\right)=\lim _{\left(t \rightarrow n T^{+}\right)} X(n T)$ exists.

(2) The smoothness properties of $f$ guarantee the global existence and uniqueness of solutions of system (1.1) (for details, see [18, 19]).

Because $x^{\prime}(t)=y^{\prime}(t)=z^{\prime}(t)=0$ whenever $x(t)=y(t)=z(t)=0 \quad(t \neq n T)$ and $z\left(n T^{+}\right)=$ $(1-\delta) z(n T)+p$, the following lemma can be stated.

Lemma 2.3. Assume $X(t)$ is a solution of system (1.1) such that

(1) if $X\left(0^{+}\right) \geq 0$, then $X(t) \geq 0$ for all $t \geq 0$,

(2) if $X\left(0^{+}\right)>0$, then $X(t)>0$ for all $t>0$.

Next, an important comparison theorem on impulsive differential equations [18] will be stated.

Lemma 2.4 (comparison theorem). Assume that $V \in V_{0}$ and that

$$
\begin{array}{cl}
D^{+} V(t, X) \leq g(t, V(t, X)), & t \neq n T, \\
V\left(t, X\left(t^{+}\right)\right) \leq \varphi_{n}(V(t, X)), & t=n T,
\end{array}
$$


where $g: R_{+} \times R_{+} \rightarrow R$ is continuous on $(n T,(n+1) T] \times R_{+}^{3}$ and for $u \in R_{+}, n \in N$, $\lim _{(t, y) \rightarrow\left(n T^{+}, u\right)} g(t, v)=g\left(n T^{+}, u\right)$ exists, with $\varphi_{n}: R_{+} \rightarrow R_{+}$nondecreasing. Let $r(t)$ be the maximal solution of the scalar impulsive differential equation:

$$
\begin{aligned}
& \frac{d u(t)}{d t}=g(t, u(t)), \quad t \neq n T, \\
& u\left(t^{+}\right)=\varphi_{n}(u(t)), \quad t=n T, \\
& u\left(0^{+}\right)=u_{0},
\end{aligned}
$$

existing on $[0, \infty)$. Then $V\left(0^{+}, X_{0}\right) \leq u_{0}$, which implies that $V(t, X(t)) \leq r(t), t \geq 0$, where $X(t)$ is any solution of system (1.1).

If the cyanobacteria and green algae are eradicated, then system (1.1) will reduce to the following system:

$$
\begin{aligned}
\frac{d z(t)}{d t} & =-m z(t), \quad t \neq n T, \\
z\left(t^{+}\right) & =(1-\delta) z(t)+p, \quad t=n T, \\
z\left(0^{+}\right) & =z_{0} .
\end{aligned}
$$

Therefore, the following result can be easily obtained.

Lemma 2.5. $z^{*}(t)=p \exp (-m(t-n T)) /(1-(1-\delta) \exp (-m T))$ is a positive periodic solution of (2.4) with initial value $z^{*}\left(0^{+}\right)=p /(1-(1-\delta) \exp (-m T)), t \in(n T,(n+1) T], n \in N$.

$z(t)=\left(z\left(0^{+}\right)-p /(1-(1-\delta) \exp (-m T))\right) \exp (-m t)+z^{*}(t)$ is a general solution of $(2.4)$ with initial value $z_{0}=z\left(0^{+}\right) \geq 0$, where $t \in(n T,(n+1) T], n \in N$.

For a positive periodic solution $z^{*}(t)$ of (2.4) and every solution $z(t)$ of $(2.4)$ with $z_{0} \geq 0$, $\left|z(t)-z^{*}(t)\right| \rightarrow 0, t \rightarrow \infty$.

From Lemma 2.5, the general solution $z(t)$ converges to the positive periodic solution $z^{*}(t)$ of (2.4) when $t \rightarrow \infty$, and the complete expression for the cyanobacteria and green algae-eradication periodic solution $\left(0,0, z^{*}(t)\right)$ of system (1.1) becomes

$$
\left(0,0, z^{*}(t)\right)=\left(0,0, \frac{p \exp (-m(t-n T))}{1-(1-\delta) \exp (-m T)}\right)
$$

for $t \in(n T,(n+1) T]$.

Now, the Floquet theorem for impulsive equations [20] will be introduced:

$$
\begin{aligned}
& \frac{d x(t)}{d t}=A(t) x(t), \quad t \neq t_{k}, \\
& x\left(t^{+}\right)=x(t)+B_{k} x(t), \quad t=t_{k},
\end{aligned}
$$


with conditions as follows

(1) $A(\cdot) \in \mathrm{PC}\left(R, C^{n \times n}\right)$ and $A(t+T)=A(t)(t \in R)$, where $\operatorname{PC}\left(R, C^{n \times n}\right)$ is the set of all piecewise continuous matrix functions and $C^{n \times n}$ is the set of $n \times n$ matrices.

(2) $B_{k} \in\left(C^{n \times n}\right)$ and $\operatorname{det}\left(E+B_{k}\right) \neq 0, t_{k} \leq T_{k+1}$.

(3) There exists a $q \in N$ such that $B_{k+q}=B_{k}, t_{k+q}=t_{k}+T$.

Let $H(t)$ be a fundamental matrix of system (2.6), then there exists a unique nonsingular matrix $M \in C^{n \times n}$ such that $H(t+T)=H(t) M . M$ is similar to the matrix of system (2.6) and has the same eigenvalues $u_{1}, u_{2}, \ldots, u_{n}$. These eigenvalues $u_{1}, u_{2}, \ldots, u_{n}$ of system (2.6) are called Floquet multipliers.

Lemma 2.6 (Floquet theorem [20]). Assume that (1)-(3) hold, then system (2.6) is

(1) stable if and only if $u_{i} \leq 1(i=1, \ldots, n)$, and to those $u_{i}=1$, there correspond simple elementary divisors;

(2) asymptotically stable if and only if all $u_{i}<1(i=1, \ldots, n)$;

(3) unstable if $u_{i}>1$ for some $i=1, \ldots, n$.

After these preliminaries, the main theorems of this paper will now be presented, then it will be proven that each solution of system (1.1) is ultimately bounded.

Theorem 2.7. There exists a positive constant $M$ such that $x(t) \leq M, y(t) \leq M$, and $z(t) \leq M$ for each solution of system (1.1) with all $t$ large enough.

Proof. Define $V(t, x(t))$ such that

$$
V(t, x(t))=x(t)+u_{2} y(t)+u_{1} z(t)
$$

Because $(d x(t) / d t) \leq r_{1} x(t)-c_{1} x^{2}(t)$, then $x(t) \leq\left(r_{1} / c_{1}\right)$, and the upper right derivative of $V(t, x(t))$ can be calculated along a solution of system (1.1) when $t \neq n T$ :

$$
\begin{aligned}
D^{+}(V(t))+L V(t) \leq & \left(L+r_{1}\right) x(t)-c_{1} x^{2}(t)+\left(L u_{2}+u_{2} r_{2}\right) y(t) \\
& -c_{2} u_{2} y^{2}(t)+\left(L u_{1}-u_{1} m+\frac{u_{1} r_{1} u_{3}}{b_{1} c_{1}}\right) z(t)
\end{aligned}
$$

Let $0<L<m-\left(r_{1} u_{3} / b_{1} c_{1}\right)$, then $\left(L+r_{1}\right) x(t)-c_{1} x^{2}(t),\left(L u_{2}+u_{2} r_{2}\right) y(t)-c_{2} u_{2} y^{2}(t)$ are both bounded, so $D^{+}(V(t))+L V(t)$ is bounded, and $L_{1}$ and $L_{2}$ can be chosen such that

$$
\begin{aligned}
\frac{d D^{+}(V(t))}{d t} & \leq-L_{1} V(t)+L_{2}, \quad t \neq n T \\
V\left(t^{+}\right) & \leq V(t)+u_{1} p, \quad t=n T
\end{aligned}
$$

where $L_{1}$ and $L_{2}$ are positive constants. According to Lemma 2.4,

$$
V(t) \leq\left(V\left(0^{+}\right)-\frac{L_{2}}{L_{1}}\right) \exp \left(-L_{1} t\right)+\frac{u_{1} p\left(1-\exp \left(-L_{1} n T\right)\right)}{1-\exp \left(-L_{1} T\right)} \exp \left(-L_{1}(t-n T)\right)+\frac{L_{2}}{L_{1}},
$$


and hence

$$
V(t) \leq \frac{L_{2}}{L_{1}}+\frac{u_{1} p \exp \left(L_{1} T\right)}{\exp \left(L_{1} T\right)-1}
$$

as $t \rightarrow \infty$, so $V(t, x(t))$ is ultimately bounded. Therefore, each positive solution of system (1.1) is ultimately bounded, and the proof is completed.

Theorem 2.8. If

$$
\begin{aligned}
\frac{r_{1} G_{0}}{G_{1}} T & <\frac{u_{1} p(1-\exp (-m T))}{m b_{1}(1-(1-\delta) \exp (-m T))}, \\
r_{2} T & <\frac{u_{1} a p(1-\exp (-m T))}{m b_{2}(1-(1-\delta) \exp (-m T))},
\end{aligned}
$$

then the periodic solution $\left(0,0, z^{*}(t)\right)$ is locally asymptotically stable.

Proof. The local stability of the periodic solution $\left(0,0, z^{*}(t)\right)$ may be determined by considering the behavior of small-amplitude perturbations of the solution.

Define $x(t)=u(t), y(t)=v(t), z(t)=w(t)+z^{*}(t)$.

Then the linearization of system (1.1) becomes

$$
\begin{aligned}
\frac{d u(t)}{d t} & =\left(\frac{r_{1} G_{0}}{G_{1}}-\frac{u_{1} z^{*}(t)}{b_{1}}\right) u(t), \\
\frac{d v(t)}{d t} & =\left(r_{2}-\frac{u_{1} a z^{*}(t)}{b_{2}}\right) v(t), \quad t \neq n T, \\
\frac{d w(t)}{d t} & =\frac{u_{3} z^{*}(t)}{b_{1}} u(t)+\frac{u_{2} a z^{*}(t)}{b_{2}} v(t)-m w(t), \\
\Delta u(t) & =0, \\
\Delta v(t) & =-\delta v(t), \quad t=n T, \\
\Delta w(t) & =0
\end{aligned}
$$

and, as a result,

$$
\left(\begin{array}{c}
u(t) \\
v(t) \\
w(t)
\end{array}\right)=\Phi(t)\left(\begin{array}{c}
u(0) \\
v(0) \\
w(0)
\end{array}\right), \quad 0 \leq t \leq T,
$$


where $\Phi(t)$ satisfies

$$
\frac{d \Phi(t)}{d t}=\left(\begin{array}{ccc}
\frac{r_{1} G_{0}}{G_{1}}-\frac{u_{1} z^{*}(t)}{b_{1}} & 0 & 0 \\
0 & r_{2}-\frac{u_{1} a z^{*}(t)}{b_{2}} & 0 \\
\frac{u_{3} z^{*}(t)}{b_{1}} & \frac{u_{2} a z^{*}(t)}{b_{2}} & -m
\end{array}\right) \Phi(t)
$$

$\Phi(0)=I$, the identity matrix, and

$$
\left(\begin{array}{c}
u\left(n T^{+}\right) \\
v\left(n T^{+}\right) \\
w\left(n T^{+}\right)
\end{array}\right)=\left(\begin{array}{ccc}
1 & 0 & 0 \\
0 & 1 & 0 \\
0 & 0 & 1-\delta
\end{array}\right)\left(\begin{array}{c}
u(n T) \\
v(n T) \\
w(n T)
\end{array}\right) .
$$

Therefore, the stability of the periodic solution $\left(0,0, z^{*}(t)\right)$ is determined by the eigenvalues

$$
\theta=\left(\begin{array}{ccc}
1 & 0 & 0 \\
0 & 1 & 0 \\
0 & 0 & 1-\delta
\end{array}\right) \Phi(T)
$$

Therefore, all eigenvalues of $\theta$ are given by

$$
\begin{aligned}
& \lambda_{1}=\exp \left(\int_{0}^{T}\left(\frac{r_{1} G_{0}}{G_{1}}-\frac{u_{1} z^{*}(t)}{b_{1}}\right) d t\right), \\
& \lambda_{2}=\exp \left(\int_{0}^{T}\left(r_{2}-\frac{u_{1} a z^{*}(t)}{b_{2}}\right) d t\right), \\
& \lambda_{3}=(1-\delta) \exp (-m T)<1 .
\end{aligned}
$$

According to Lemma 2.6, $\left(0,0, z^{*}(t)\right)$ is locally asymptotically stable if $\lambda_{1}<1, \lambda_{2}<1$, that is to say

$$
\begin{aligned}
\frac{r_{1} G_{0}}{G_{1}} T & <\frac{u_{1} p(1-\exp (-m T))}{m b_{1}(1-(1-\delta) \exp (-m T))}, \\
r_{2} T & <\frac{u_{1} a p(1-\exp (-m T))}{m b_{2}(1-(1-\delta) \exp (-m T))},
\end{aligned}
$$

which completes the proof. 
Theorem 2.9. If

$$
\begin{gathered}
\frac{r_{1} G_{0}}{G_{1}} T<\frac{u_{1} p(1-\exp (-m T))}{m b_{1}(1-(1-\delta) \exp (-m T))}, \\
r_{2} T<\frac{u_{1} a p(1-\exp (-m T))}{m b_{2}(1-(1-\delta) \exp (-m T))}, \\
r_{1}-\frac{u_{1}}{b_{1}+M}\left(\frac{p \exp (-m T)}{1-(1-\delta) \exp (-m T)}\right)<0 \\
r_{2}-\frac{u_{1} a}{M+a M+b_{2}}\left(\frac{p \exp (-m T)}{1-(1-\delta) \exp (-m T)}\right)<0
\end{gathered}
$$

then the periodic solution $\left(0,0, z^{*}(t)\right)$ is globally asymptotically stable.

Proof. From Theorem 2.8 , the periodic solution $\left(0,0, z^{*}(t)\right)$ is locally asymptotically stable. Now it will be proved to be a global attractor. Let $V(t)=x(t)+y(t)$, then by Theorem 2.7, there exists a constant $M>0$ such that $x(t) \leq M, y(t) \leq M$, and $z(t) \leq M$. Obviously,

$$
\left.V^{\prime}\right|_{(1.1)} \leq x(t)\left(r_{1}-\frac{u_{1} z(t)}{b_{1}+M}\right)-c_{1} x^{2}(t)+y(t)\left(r_{2}-\frac{u_{1} a z(t)}{M+a M+b_{2}}\right)-c_{2} y^{2}(t)
$$

However, for system (1.1),

$$
\begin{aligned}
& \frac{d z(t)}{d t} \geq-m z(t), \quad t \neq n T, \\
& z\left(t^{+}\right)=(1-\delta) z(t)+p, \quad t=n T .
\end{aligned}
$$

Therefore, there exists a $\tau>0$, and an $\varepsilon>0$ small enough, such that $z(t) \geq z_{1}^{*}(t)-\varepsilon$ for all $t>\tau$. This leads to

$$
z(t) \geq z_{1}^{*}(t)-\varepsilon=\frac{p \exp (-m T)}{1-(1-\delta) \exp (-m T)}-\varepsilon
$$

Let $\gamma \triangleq p \exp (-m T) /(1-(1-\delta) \exp (-m T))-\varepsilon$; it is well known that $r_{1}-\left(u_{1} \gamma /\left(b_{1}+M\right)\right)<$ 0 and $r_{2}-\left(u_{1} a r /\left(M+a M+b_{2}\right)\right)<0$. Therefore, when $t>\tau$,

$$
\left.V^{\prime}\right|_{(1.1)} \leq x(t)\left(r_{1}-\frac{u_{1} z(t)}{b_{1}+M}\right)-c_{1} x^{2}(t)+y(t)\left(r_{2}-\frac{u_{1} a z(t)}{M+a M+b_{2}}\right)-c_{2} y^{2}(t)<0
$$

Therefore, $V(t) \rightarrow 0, x(t) \rightarrow 0, y(t) \rightarrow 0$ as $t \rightarrow \infty$, and it follows that the periodic solution $\left(0,0, z^{*}(t)\right)$ is a global attractor. This completes the proof. 
Theorem 2.10. The system (1.1) is permanent, if

$$
\begin{gathered}
\frac{r_{1} G_{0}}{G_{1}} T>\frac{u_{1} p(1-\exp (-m T))}{m b_{1}(1-(1-\delta) \exp (-m T))}, \\
r_{2} T \frac{u_{1} a p(1-\exp (-m T))}{m b_{2}(1-(1-\delta) \exp (-m T))}, \\
r_{1}-\frac{u_{1}}{b_{1}+M}\left(\frac{p \exp (-m T)}{1-(1-\delta) \exp (-m T)}\right)>0, \\
r_{2}-\frac{u_{1} a}{M+a M+b_{2}}\left(\frac{p \exp (-m T)}{1-(1-\delta) \exp (-m T)}\right)>0 .
\end{gathered}
$$

Proof. Assume that $X(t)=(x(t), y(t), z(t))$ is any solution of system (1.1) with $X(0)>0$. From Theorem 2.7, $x(t) \leq M, y(t) \leq M$, and $z(t) \leq M$ with $t \geq 0$. From Lemma 2.5, $z(t) \geq z^{*}(t)-\varepsilon$ for all $t$ large enough and some $t$ with $z(t)>p \exp (-m T) /(1-(1-\delta) \exp (-m T))-\varepsilon=\xi_{1}$. Therefore, it is necessary to find $\xi_{2}$ and $\xi_{3}$ such that $x(t) \geq \xi_{2}$ and $y(t) \geq \xi_{3}$ for $t$ large enough. Select an $\varepsilon_{1}$ small enough so that

$$
\varphi_{1}=\exp \left(\int_{n T}^{(n+1) T}\left(\frac{r_{1} G_{0}}{G_{1}}-\frac{r_{1} M}{G_{1}-M}-c_{1} M-\frac{u_{1}}{b_{1}}\left(v^{*}(t)+\varepsilon_{1}\right)\right) d t\right)>1 .
$$

Next, it is necessary to prove that $x(t) \geq \xi_{2}$ for $t$ large enough, where $\xi_{2}$ is a positive constant.

It is easy to prove that there exists a $t_{1} \in(0, \infty)$ such that $x(t) \geq \xi_{2}$. Otherwise, if $x(t)<\xi_{2}$ for all $t>0$,

$$
\begin{aligned}
\frac{d z(t)}{d t} & \leq\left(\frac{u_{2} a M}{b_{2}}+\frac{u_{3} M}{b_{1}}-m\right) z(t), \quad t \neq n T, \\
z\left(t^{+}\right) & =(1-\delta) z(t)+p, \quad t=n T, \\
z\left(0^{+}\right) & =z_{0},
\end{aligned}
$$

and therefore $z(t) \leq v(t)$ and $v(t) \rightarrow v^{*}(t)$, where $v(t)$ is the solution of

$$
\begin{aligned}
\frac{d v(t)}{d t} & =\left(\frac{u_{2} a M}{b_{2}}+\frac{u_{3} M}{b_{1}}-m\right) v(t), \quad t \neq n T, \\
v\left(t^{+}\right) & =(1-\delta) v(t)+p, \quad t=n T, \\
v\left(0^{+}\right) & =v_{0},
\end{aligned}
$$

with

$$
v^{*}(t)=\frac{p \exp \left(-\left(m-\left(u_{2} a M / b_{2}\right)-\left(u_{3} M / b_{1}\right)\right)(t-n T)\right)}{1-(1-\delta) \exp \left(-\left(m-\left(u_{2} a M / b_{2}\right)-\left(u_{3} M / b_{1}\right)\right) T\right)}, \quad t \in(n T,(n+1) T] .
$$


Therefore, there exists a $T_{1}>0$ such that $z(t) \leq v(t) \leq z^{*}(t)+\varepsilon_{1}$, and

$$
\frac{d x(t)}{d t} \geq x(t)\left[\frac{r_{1} G_{0}}{G_{1}}-\frac{r_{1} M}{G_{1}-M}-c_{1} M-\frac{u_{1}}{b_{1}}\left(v^{*}(t)+\varepsilon_{1}\right)\right],
$$

for $t>T_{1}$. Let $N_{1} \in N$ and $N_{1} T \geq T_{2} \geq T_{1}$. Integrating (2.30) over $(n T,(n+1) T] n>N_{1}$ yields

$$
\begin{gathered}
x((n+1) T) \geq x\left(n T^{+}\right) \exp \left(\int_{n T}^{(n+1) T}\left(\frac{r_{1} G_{0}}{G_{1}}-\frac{r_{1} M}{G_{1}-M}-c_{1} M-\frac{u_{1}}{b_{1}}\left(v^{*}(t)+\varepsilon_{1}\right)\right) d t\right)=x\left(n T^{+}\right), \\
\exp \left(\int_{n T}^{(n+1) T}\left(\frac{r_{1} G_{0}}{G_{1}}-\frac{r_{1} M}{G_{1}-M}-c_{1} M-\frac{u_{1}}{b_{1}}\left(v^{*}(t)+\varepsilon_{1}\right)\right) d t\right)=x\left(n T^{+}\right) \varphi_{1} .
\end{gathered}
$$

Therefore, $x\left(\left(N_{1}+n\right) T\right) \geq x\left(N_{1} T\right) \varphi_{1}^{n} \rightarrow \infty$ as $n \rightarrow \infty$, but this leads to a contradiction because $x(t)$ is bounded. Therefore, there exists a $t_{1}>0$ such that $x\left(t_{1}\right) \geq \xi_{2}$.

Second, if $x\left(t_{1}\right) \geq \xi_{2}$ for all $t \geq t_{1}$, then the proof is complete. Hence, it is only necessary to consider those solutions which leave the region $R=\left\{x(t): x(t)<\xi_{2}\right\}$ and reenter it. Let $t^{*}(t)=\inf _{t \geq t_{1}}\left\{x(t)<\xi_{2}\right\}$, then $x(t) \geq \xi_{2}, t \in\left(t, t^{*}\right)$, and $t^{*} \in\left(n_{1} T,\left(n_{1}+1\right) T\right)$. However, obviously $x\left(t^{*}\right)=\xi_{2}$ because $X(t)=(x(t), y(t), z(t))$ is continuous. It is claimed here that there must exist a $t_{2} \in\left(\left(n_{1}+1\right) T,\left(n_{1}+1\right) T+T_{1}\right)$ such that $x\left(t_{2}\right) \geq \xi_{2}$. Otherwise, $x(t)<\xi_{2}$, for $t \in\left(\left(n_{1}+1\right) T,\left(n_{1}+1\right) T+T_{1}\right), T_{1}=n_{2} T+n_{3} T, n_{2}, n_{3} \in N$, and then

$$
\begin{gathered}
\left(n_{2}-1\right) T>\frac{\ln \left(\varepsilon_{1} /(M+p)\right)}{-\left(\left(u_{2} a M / b_{2}\right)+\left(u_{3} M / b_{1}\right)-m\right)}, \\
\exp \left(\varphi_{2}\left(n_{2}+1\right) T\right) \varphi_{1}^{n_{3}}>1 .
\end{gathered}
$$

Consider (2.28) with $v\left(t^{*}+\right)=z\left(t^{*}+\right)$; this leads to

$$
\begin{aligned}
v(t)= & \left(v\left(\left(n_{1}+1\right) T^{+}\right)-\frac{p}{1-(1-\delta) \exp \left(-\left(\left(u_{2} a M / b_{2}\right)+\left(u_{3} M / b_{1}\right)-m\right) T\right)}\right) \\
& \times \exp \left(-\left(\frac{u_{2} a M}{b_{2}}+\frac{u_{3} M}{b_{1}}-m\right)\left(t-\left(n_{1}+1\right) T\right)\right)+v^{*}(t),
\end{aligned}
$$

for $t \in(n T,(n+1) T], n_{1}+1<n<n_{1}+n_{2}+n_{3}+1$, and then

$$
\begin{gathered}
\left|v(t)-v^{*}(t)\right|<(M+p) \exp \left(-\left(\frac{u_{2} a M}{b_{2}}+\frac{u_{3} M}{b_{1}}-m\right)\left(t-\left(n_{1}+1\right) T\right)\right)<\varepsilon_{1}, \\
z(t) \leq v(t) \leq v^{*}(t)+\varepsilon_{1}
\end{gathered}
$$

for $t \in\left[\left(n_{1}+n_{2}+1\right) T,\left(n_{1}+1\right) T+T_{1}\right]$, which implies (2.30). Integrating (2.30) over $t \in\left[\left(n_{1}+\right.\right.$ $\left.\left.n_{2}+1\right) T,\left(n_{1}+1\right) T+T_{1}\right]$ yields

$$
x\left(\left(n_{1}+n_{2}+n_{3}+1\right) T\right) \geq x\left(\left(n_{1}+n_{2}+1\right) T\right) \varphi_{1}^{n_{3}} .
$$


For every $t \in\left(t^{*},\left(n_{1}+1\right) T\right)$, there are only two possible cases: one, if $x(t)<\xi_{2}$ for $t \in\left(t^{*},\left(n_{1}+1\right) T\right)$, when $t \in\left(t^{*},\left(n_{1}+n_{2}+1\right) T\right)$, then $x(t)<\xi_{2}$, and hence

$$
\frac{d x(t)}{d t} \geq x(t)\left[\frac{r_{1} G_{0}}{G_{1}}-\frac{r_{1} \xi_{2}}{G_{1}-M}-c_{1} M-\frac{u_{1}}{b_{1}} M\right]=\varphi_{2} x(t) .
$$

Integrating (2.36) over $t \in\left(t^{*},\left(n_{1}+n_{2}+1\right) T\right), x\left(\left(n_{1}+n_{2}+1\right) T\right) \geq x\left(t^{*}\right) \exp \left(\varphi_{2}\left(n_{2}+1\right) T\right)$ is obvious.

Therefore, $x\left(\left(n_{1}+n_{2}+n_{3}+1\right) T\right) \geq \xi_{2} \exp \left(\varphi_{2}\left(n_{2}+1\right) T\right) \varphi_{1}^{n_{3}}>\xi_{2}$, but this leads to a contradiction. Hence, let $t_{3}=\inf _{t>t^{*}}\left\{x(t) \geq \xi_{2}\right\}$, then $x\left(t_{3}\right)=\xi_{2}$, and (2.36) holds. Integrating (2.36) over $\left[t^{*}, t_{3}\right)$,

$$
x(t) \geq x\left(t^{*}\right) \exp \left(\varphi_{2}\left(t-t^{*}\right)\right) \geq \xi_{2} \exp \left(\varphi_{2}\left(n_{1}+n_{3}+1\right) T\right)=\xi_{5} .
$$

$x\left(t_{3}\right) \geq \xi_{2}$ is also true for $t>t_{3}$. Hence, $x(t) \geq \xi_{5}, t>t_{3}$. Two, there exists a $t_{5} \in$ $\left(t^{*},\left(n_{1}+1\right) T\right]$ such that $x\left(t_{5}\right) \geq \xi_{2}$. Therefore, let $t_{4}=\inf _{t>t^{*}}\left\{x(t) \geq \xi_{2}\right\}$, then $x(t)<\xi_{2}$ for $t \in\left[t^{*}, t_{4}\right)$ and $x\left(t_{4}\right)=\xi_{2}$. For $t \in\left[t^{*}, t_{4}\right),(2.36)$ holds, and integrating it over $t \in\left[t^{*}, t_{4}\right)$, $x(t) \geq x\left(t^{*}\right) \exp \left(\varphi_{2}\left(t-t^{*}\right)\right)>\xi_{5}$. This process can be continued because $x\left(t_{4}\right) \geq \xi_{2}$ and $x(t) \geq$ $\xi_{5}, t>t_{4}$. This yields the two possible cases, $x(t) \geq \xi_{5}, t \geq t_{1}$, and $y(t) \geq \xi_{4}, t \geq t_{2}$. Let $\Omega=\left\{(x, y, z): x(t) \geq \xi_{2}, y(t) \geq \xi_{3}, z(t) \geq \xi_{1}, x(t)+y(t)+z(t) \leq 3 M\right\}$. Then the set $\Omega$ is a global attractor. Moreover, every solution $X(t)=(x(t), y(t), z(t))$ of system (1.1) will eventually enter and remain in the set $\Omega$. Therefore, system (1.1) is permanent, and the proof is completed.

\section{Numerical Analysis}

\subsection{Bifurcation Analysis}

In Section 2, it has been proved that the cyanobacteria and green algae-eradication periodic solution is locally asymptotically stable, and conditions have been established for the permanence of system (1.1). Now, to substantiate these theoretical results, the following parameters will be considered: $u_{1}=0.5, u_{2}=0.6, u_{3}=0.4, r_{1}=0.5, r_{2}=0.5, G_{0}=10, G_{1}=15$, $y_{m}=15, y_{n}=20, c_{1}=0.1, c_{2}=0.12, a=0.75, b_{1}=0.45, b_{2}=0.6, \delta=0.55, T=40$ with initial values $x_{0}=0.5, y_{0}=0.6, z_{0}=0.7$.

From Theorem 2.8, it is known that the periodic solution $\left(0,0, z^{*}(t)\right)$ is locally asymptotically stable if $p>p_{\max }=8.15$; this periodic solution $\left(0,0, z^{*}(t)\right)$ is shown in Figure 1 . It is clear that the variable fish $z$ oscillates in a stable cycle, but the cyanobacteria population $x$ and green algae population $y$ rapidly decrease to zero when $p>p_{\max }$. When $p$ is smaller than $p_{\max }$, then the cyanobacteria and green algae-eradication periodic solution will become unstable, and it is possible that the cyanobacteria population, green algae population, and fish population can coexist.

Figure 2 shows typical bifurcation diagrams for system (1.1) with respect to $p$ in the range $p \in[0,10]$. As $p$ increases, system (1.1) exhibits rich dynamic behavior, such as chaotic bands with periodic windows, crises, period-halving bifurcations, quasi-periodic oscillations, and narrow or wide periodic windows. When $p<0.89$, the cyanobacteria population $x$ enters a chaotic band. As $p$ increases beyond 0.89 , the cyanobacteria population 


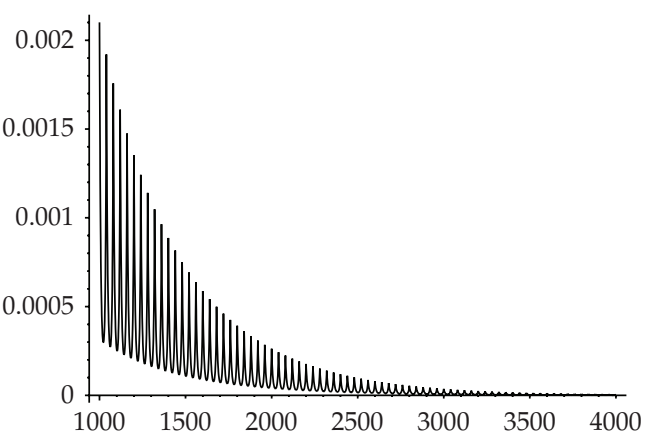

(a)

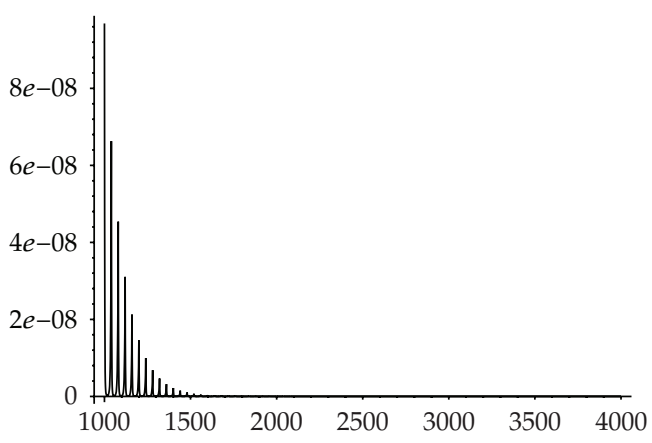

(b)

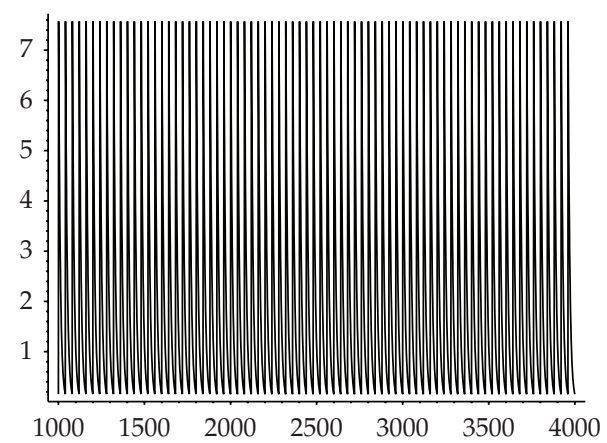

(c)

Figure 1: Dynamic behavior of system (1.1). When $p>p_{\max }=8.15$, the cyanobacteria and green algae will become extinct. Time series evolving according to biological control system (1.1): (a) the cyanobacteria population $x,(\mathrm{~b})$ the green algae population $y$, and (c) the fish population $z$.

$x$ enters a chaotic band with periodic windows, crisis phenomena appear, and the chaotic attractor suddenly changes into a periodic attractor. Then the periodic attractor loses its stability, and system (1.1) again enters a chaotic band. Finally, the chaotic attractor changes into a periodic attractor again; details of these results are shown in Figure 3. When $1.15<$ $p<2.7$, there is a cascade of period-halving bifurcation which leads system (1.1) to a $T$-periodic solution (Figure 4), where the period-halving bifurcation is the opposite of the bifurcation observed earlier. When $p>2.75$, the green algae population $y$ decreases to zero because of the increasing number of fish population $z$ and the competition between the cyanobacteria population $x$ and green algae population $y$. Then the cyanobacteria population $x$ and fish population $z$ can coexist in a stable cycle, but the numbers of the cyanobacteria population $x$ will decrease because of short supply of resources. When $p>p_{\max }=8.15$ the cyanobacteria population $x$ will be eradicated because the number of fish population $z$ released is continually increasing, then the cyanobacteria and green algae-eradication periodic solution occurs. Once the cyanobacteria population $x$ and green algae population $y$ have decreased to zero, fish population $z$ will be eradicated after a period of time because of lack of food. Therefore, it is apparent that the value of $p$ can effectively control the size of the cyanobacteria population and green algae population. All these numerical simulations are consistent with the theoretical proofs presented earlier. 


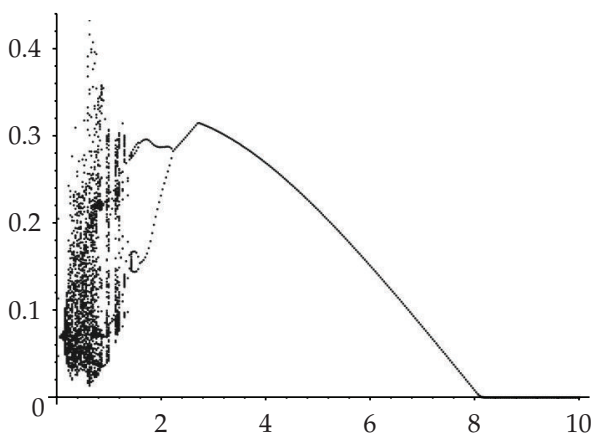

(a)

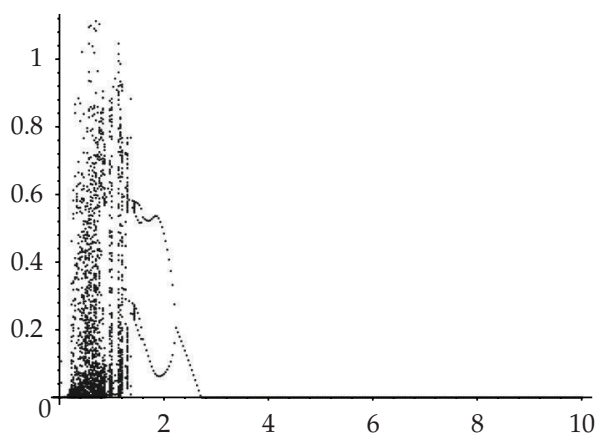

(b)

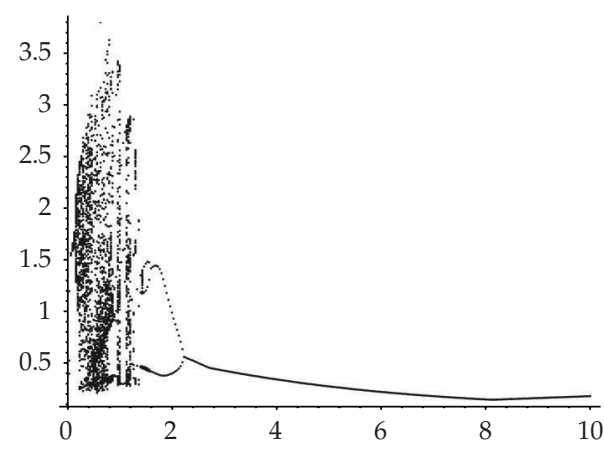

(c)

Figure 2: Bifurcation diagrams for system (1.1) showing the effect of $p$. (a) $x$ versus $p$, (b) $y$ versus $p$, and (c) $z$ versus $p$.

\subsection{Strange Attractors and Power Spectra}

Now the commonly used method called power spectra will be used to study the qualitative nature of strange attractors [21]. By calculating the largest Lyapunov exponent for strange attractor (a) (Figure 5(a)), this value is determined to be 0.18219 . Obviously, the strange attractor is a chaotic attractor. Furthermore, the spectrum of chaotic attractor (a) (Figure 5(b)) is composed of dense wave bands and sparse wave peaks with low wave troughs. These results agree with the observation that the chaotic attractor comes into being because some cycles lose weak stability.

\subsection{The Largest Lyapunov Exponent}

Convincing evidence for deterministic chaos has come from several recent experiments [2224]. From these results, it is clear that chaos plays a very important role in these studies, and therefore detecting and exploring chaos is very important [25-31]. Therefore, the largest Lyapunov exponent is considered to be the most useful diagnostic tool for chaotic systems. The largest Lyapunov exponent $\lambda$ must be positive for a chaotic attractor, otherwise, if $\lambda$ is negative, the system will enter a stable state or become a periodic attractor. The largest Lyapunov exponent for system (1.1) was calculated for various values of $p$, and Figure 6 shows the results for $p$ from 0 to 2 . 


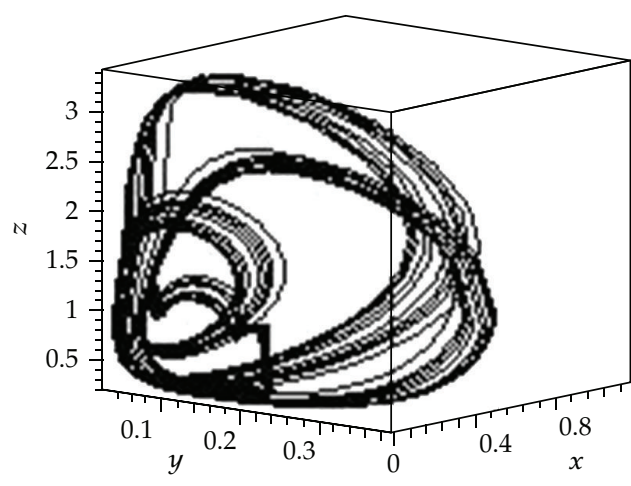

(a)

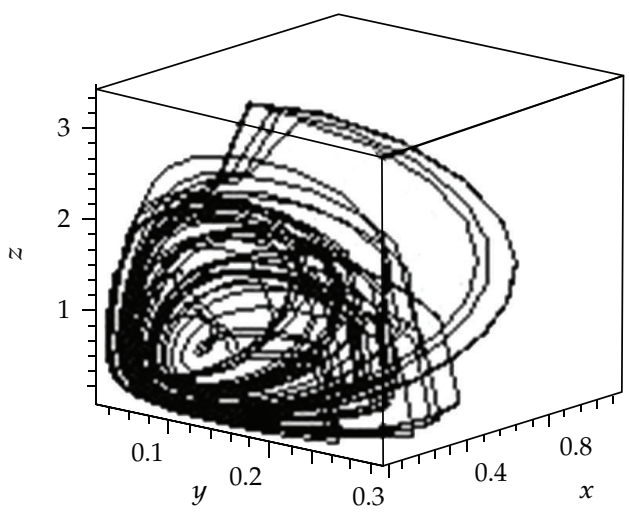

(c)

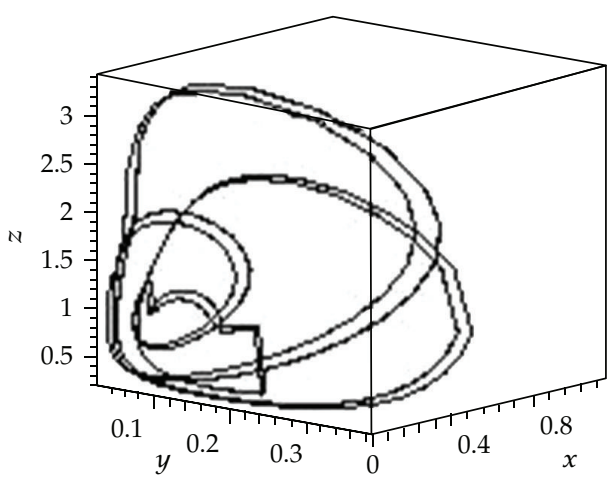

(b)

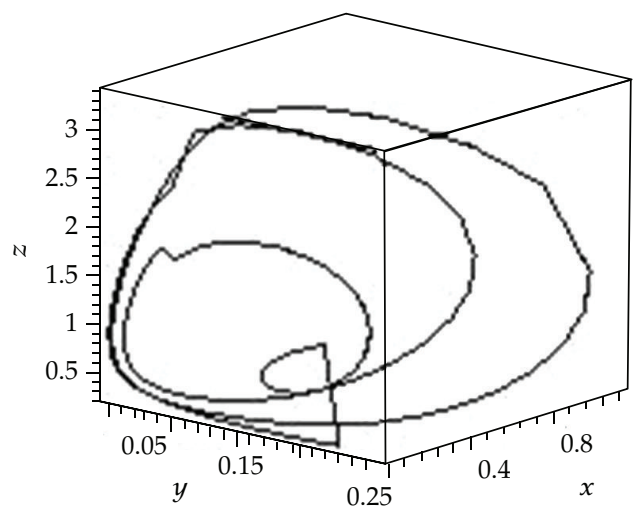

(d)

Figure 3: Crisis. (a) Chaotic attractor when $p=0.88$, (b) phase diagram of periodic solution when $p=0.9$, (c) chaotic attractor when $p=1$, and (d) phase diagram of periodic solution when $p=1.1$.

\section{Conclusions}

In this paper, the effects of impulsive perturbations on a fish algae model have been investigated. It has been proved by means of the Floquet theory of impulsive differential equations and small-amplitude perturbation techniques that system (1.1) has a stable algae-eradication periodic solution. Furthermore, the conditions for system (1.1) to be permanent have been determined using the comparison theorem. Typical bifurcation diagrams have been analyzed in detail, revealing that the system exhibits very rich dynamics. From Theorem 2.8 , it is clear that the cyanobacteria and green algae-eradication periodic solution $\left(0,0, z^{*}(t)\right)$ is locally asymptotically stable if $p>p_{\max }=8.15$, but that the cyanobacteria population $x$ and green algae population $y$ rapidly decrease to zero. When the value of $p>2.75$, green algae population $y$ decreases to zero, but the cyanobacteria population $x$ and fish population $z$ can coexist in a cycle; when $2.25<p<2.75$, the cyanobacteria population, green algae population and fish population can coexist in a cycle. When $0<p<2.25$, system (1.1) undergoes complex dynamics. From the above analysis, it is apparent that the numbers of both algae species can be controlled effectively using an impulsive control strategy. 


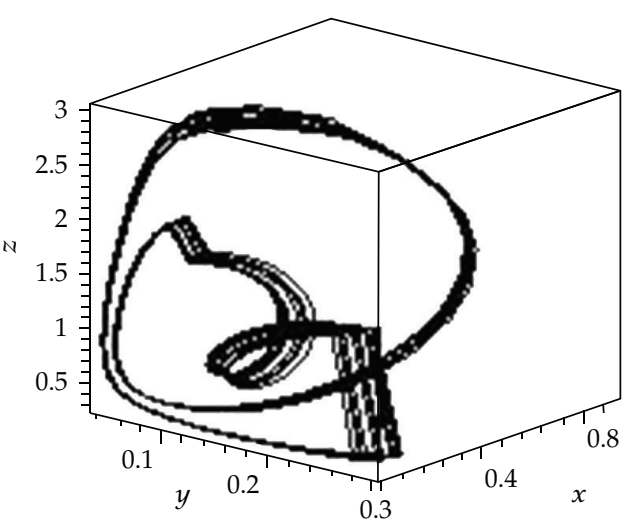

(a)

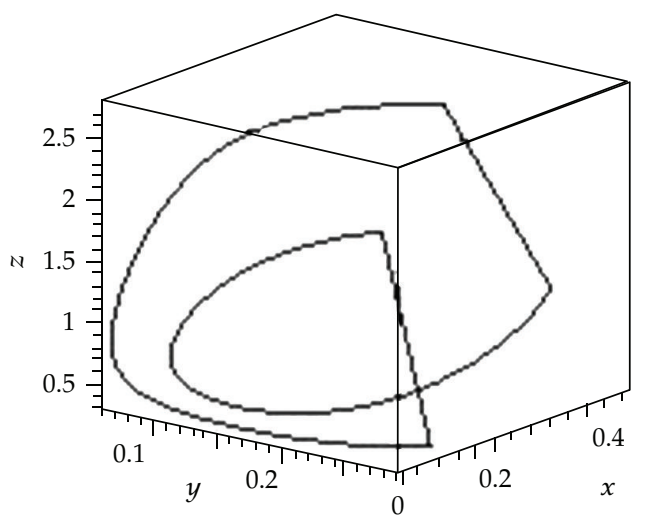

(c)

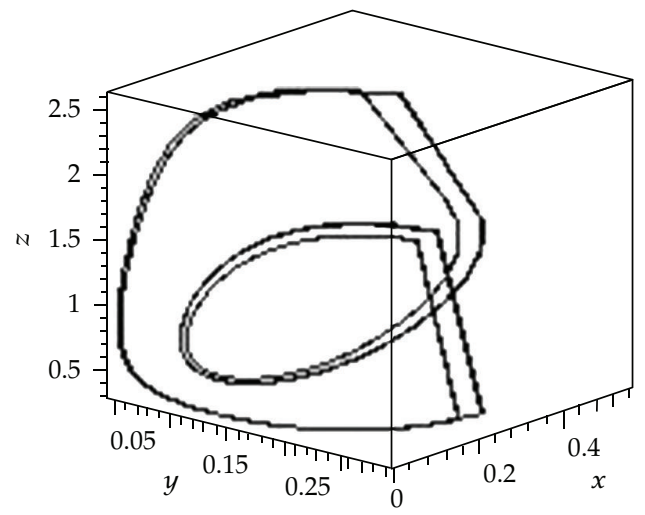

(b)

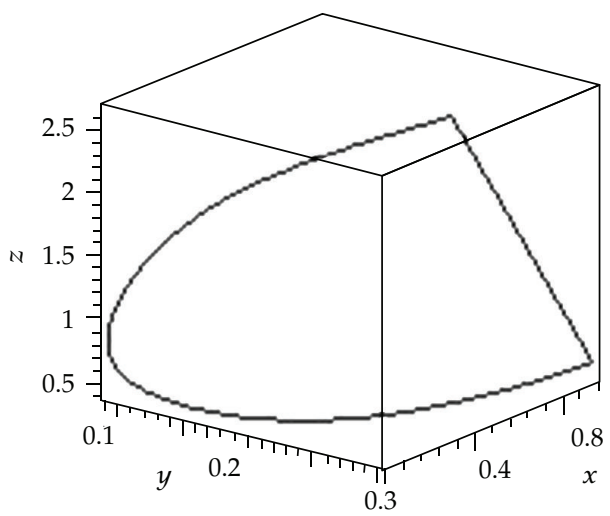

(d)

Figure 4: Period-halving bifurcation. (a) Chaotic attractor when $p=1.3$, (b) phase diagram of a 4T-periodic solution when $p=1.55$, (c) phase diagram of a 2T-periodic solution when $p=2$, and (d) a period-T attractor when $p=2.5$.

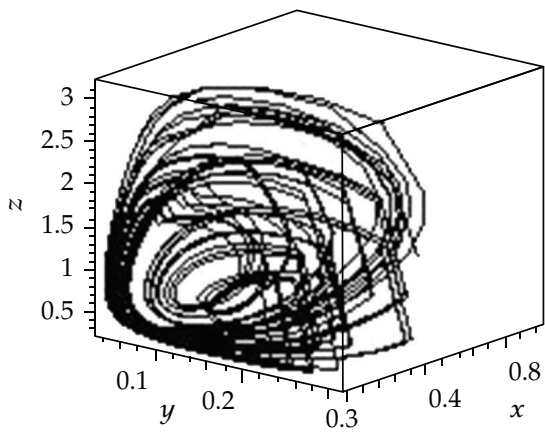

(a)

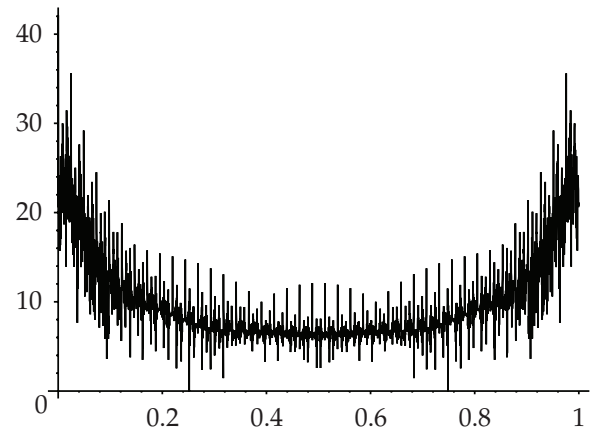

(b)

Figure 5: Strange attractor and power spectrum. (a) Strange attractor when $p=1.25$, (b) power spectrum of attractor (a). 


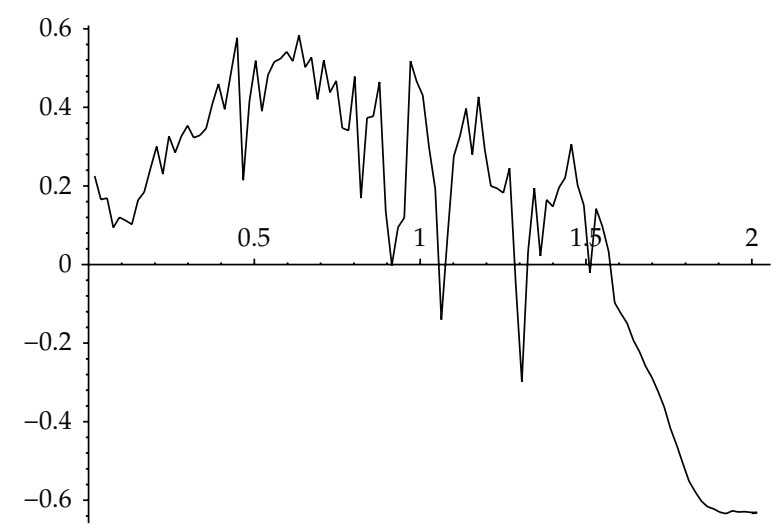

Figure 6: The largest Lyapunov exponents (LLE) for system (1.1) with $p$ varying between 0 and 2.

\section{Acknowledgments}

The authors would like to thank the editor and the anonymous referees for their valuable comments and suggestions on this paper. This work was supported by the National Natural Science Foundation of China (Grant no. 31170338 and Grant no. 30970305).

\section{References}

[1] S. E. Prochnik, J. Umen, A. M. Nedelcu et al., "Genomic analysis of organismal complexity in the multicellular green alga volvox carteri," Science, vol. 329, no. 5988, pp. 223-226, 2010.

[2] C. Ye, Q. Xu, H. Kong, Z. Shen, and C. Yan, "Eutrophication conditions and ecological status in typical bays of Lake Taihu in China," Environmental Monitoring and Assessment, vol. 135, no. 1-3, pp. 217-225, 2007.

[3] T. Y. Long, L. Wu, G. H. Meng, and W. H. Guo, "Numerical simulation for impacts of hydrodynamic conditions on algae growth in Chongqing Section of Jialing River, China," Ecological Modelling, vol. 222, no. 1, pp. 112-119, 2011.

[4] Z. L. Shen and Q. Liu, "Nutrients in the changjiang river," Environmental Monitoring and Assessment, vol. 153, no. 1-4, pp. 27-44, 2009.

[5] F. D. Parker, "Management of pest populations by manipulating densities of both host and parasites through periodic releases," in Biological Control, C. B. Huffaker, Ed., Plenum Press, New York, NY, USA, 1971.

[6] H. I. Freedman, "Graphical stability, enrichment, and pest control by a natural enemy," Mathematical Biosciences, vol. 31, no. 3-4, pp. 207-225, 1976.

[7] H. Liu, H. Xu, J. Yu, and G. Zhu, "Stability on coupling SIR epidemic model with vaccination," Journal of Applied Mathematics, vol. 2005, no. 4, pp. 301-319, 2005.

[8] R. Shi, X. Jiang, and L. Chen, "A predator-prey model with disease in the prey and two impulses for integrated pest management," Applied Mathematical Modelling. Simulation and Computation for Engineering and Environmental Systems, vol. 33, no. 5, pp. 2248-2256, 2009.

[9] F. Chen, "Periodicity in a ratio-dependent predator-prey system with stage structure for predator," Journal of Applied Mathematics, vol. 2005, no. 2, pp. 153-169, 2005.

[10] R. Xu, M. A. J. Chaplain, and F. A. Davidson, "Periodic solutions of a delayed predator-prey model with stage structure for predator," Journal of Applied Mathematics, vol. 2004, no. 3, pp. 255-270, 2004.

[11] J. Z. Farkas, "On the linearized stability of age-structured multispecies populations," Journal of Applied Mathematics, vol. 2006, Article ID 60643, 8 pages, 2006.

[12] S. A. Hadley and L. K. Forbes, "Dynamical systems analysis of a five-dimensional trophic food web model in the southern oceans," Journal of Applied Mathematics, vol. 2009, Article ID 575047, 17 pages, 2009. 
[13] L. Zhang, Z. Teng, and Z. Liu, "Survival analysis for a periodic predatory-prey model with prey impulsively unilateral diffusion in two patches," Applied Mathematical Modelling., vol. 35, no. 9, pp. 4243-4256, 2011.

[14] H. Yu, S. Zhong, and R. P. Agarwal, "Mathematics and dynamic analysis of an apparent competition community model with impulsive effect," Mathematical and Computer Modelling, vol. 52, no. 1-2, pp. 25-36, 2010.

[15] H. Yu, S. Zhong, R. P. Agarwal, and S. K. Sen, "Three-species food web model with impulsive control strategy and chaos," Communications in Nonlinear Science and Numerical Simulation, vol. 16, no. 2, pp. 1002-1013, 2011.

[16] H. Yu, S. Zhong, R. P. Agarwal, and L. Xiong, "Species permanence and dynamical behavior analysis of an impulsively controlled ecological system with distributed time delay," Computers $\mathcal{E}$ Mathematics with Applications, vol. 59, no. 12, pp. 3824-3835, 2010.

[17] X. Wang, H. Yu, S. Zhong, and R. P. Agarwal, "Analysis of mathematics and dynamics in a food web system with impulsive perturbations and distributed time delay," Applied Mathematical Modelling, vol. 34, no. 12, pp. 3850-3863, 2010.

[18] V. Lakshmikantham, D. D. Baĭnov, and P. S. Simeonov, Theory of Impulsive Differential Equations, vol. 6 of Series in Modern Applied Mathematics, World Scientific, Teaneck, NJ, USA, 1989.

[19] M. Benchohra, J. Henderson, and S. Ntouyas, Impulsive Differential Equations and Inclusions, vol. 2 of Contemporary Mathematics and Its Applications, Hindawi Publishing Corporation, New York, NY, USA, 2006.

[20] H. K. Baek, "Qualitative analysis of Beddington-DeAngelis type impulsive predator-prey models," Nonlinear Analysis: Real World Applications, vol. 11, no. 3, pp. 1312-1322, 2010.

[21] D. Baĭnov and P. Simeonov, Impulsive Differential Equations: Periodic Solutions and Applications, vol. 66 of Pitman Monographs and Surveys in Pure and Applied Mathematics, Longman Scientific \& Technical, Harlow, UK, 1993.

[22] C. Masoller, A. C. S. Schifino, and L. Romanelli, "Characterization of strange attractors of lorenz model of general circulation of the atmosphere," Chaos, Solitons E Fractals, vol. 6, pp. 357-366, 1995.

[23] F. Grond, H. H. Diebner, S. Sahle, A. Mathias, S. Fischer, and O. E. Rossler, "A robust, locally interpretable algorithm for Lyapunov exponents," Chaos, Solitons E Fractals, vol. 16, no. 5, pp. 841-852, 2003.

[24] J. C. Sprott, Chaos and Time-Series Analysis, Oxford University Press, New York, NY, USA, 2003.

[25] M. T. Rosenstein, J. J. Collins, and C. J. De Luca, "A practical method for calculating largest Lyapunov exponents from small data sets," Physica D, vol. 65, no. 1-2, pp. 117-134, 1993.

[26] M. Zhao, H. Yu, and J. Zhu, "Effects of a population floor on the persistence of chaos in a mutual interference host-parasitoid model," Chaos, Solitons \& Fractals, vol. 42, no. 2, pp. 1245-1250, 2009.

[27] L. Zhang and M. Zhao, "Dynamic complexities in a hyperparasitic system with prolonged diapause for host," Chaos, Solitons \& Fractals, vol. 42, no. 2, pp. 1136-1142, 2009.

[28] S. Gakkhar and R. K. Naji, "Order and chaos in a food web consisting of a predator and two independent preys," Communications in Nonlinear Science and Numerical Simulation, vol. 10, no. 2, pp. 105-120, 2005.

[29] S. Lv and M. Zhao, "The dynamic complexity of a three species food chain model," Chaos, Solitons $\mathcal{E}$ Fractals, vol. 37, no. 5, pp. 1469-1480, 2008.

[30] M. Zhao and L. Zhang, "Permanence and chaos in a host-parasitoid model with prolonged diapause for the host," Communications in Nonlinear Science and Numerical Simulation, vol. 14, no. 12, pp. 41974203, 2009.

[31] L. Zhu and M. Zhao, "Dynamic complexity of a host-parasitoid ecological model with the Hassell growth function for the host," Chaos, Solitons E Fractals, vol. 39, no. 3, pp. 1259-1269, 2009. 


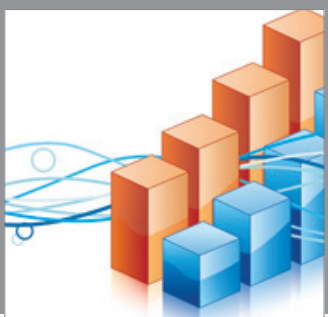

Advances in

Operations Research

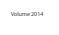

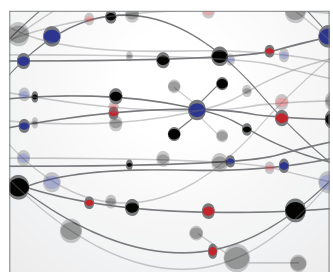

\section{The Scientific} World Journal
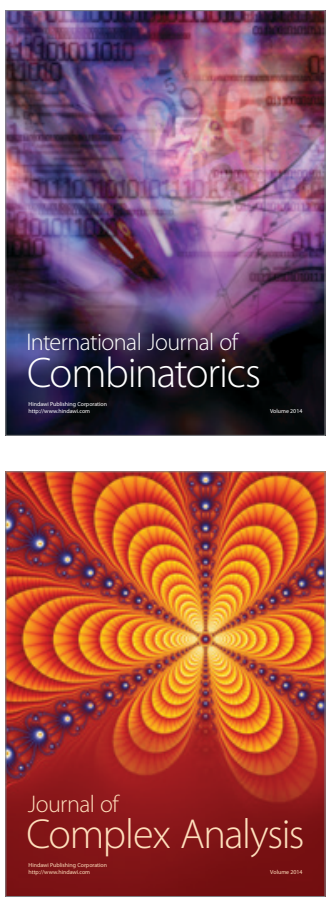

International Journal of

Mathematics and

Mathematical

Sciences
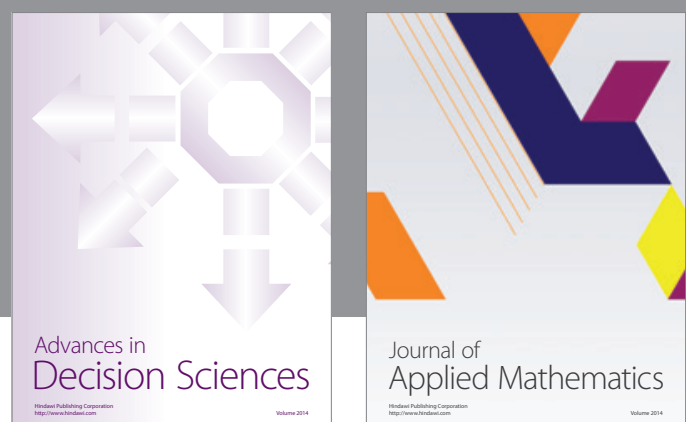

Journal of

Applied Mathematics
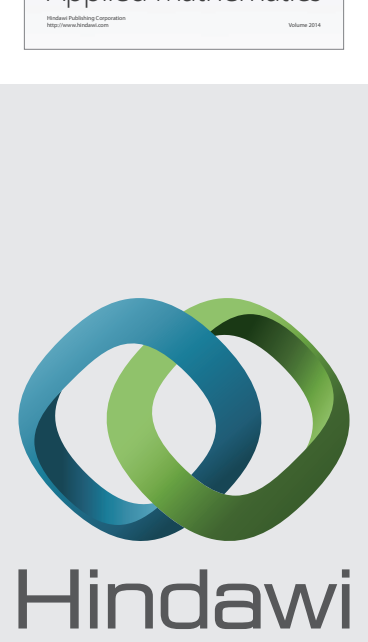

Submit your manuscripts at http://www.hindawi.com
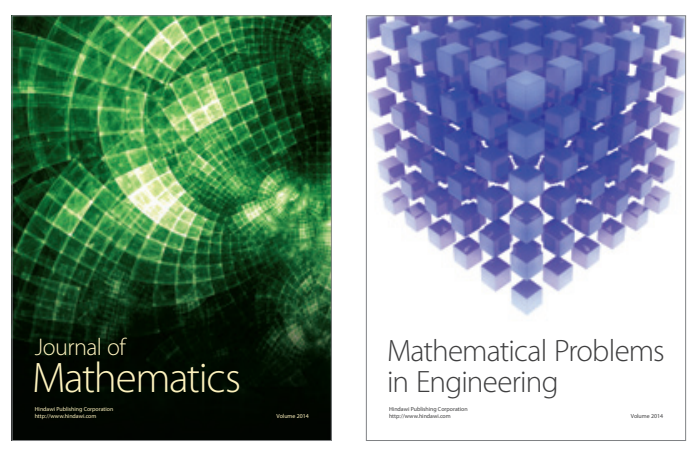

Mathematical Problems in Engineering
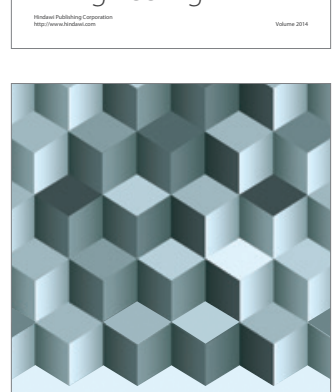

Journal of

Function Spaces
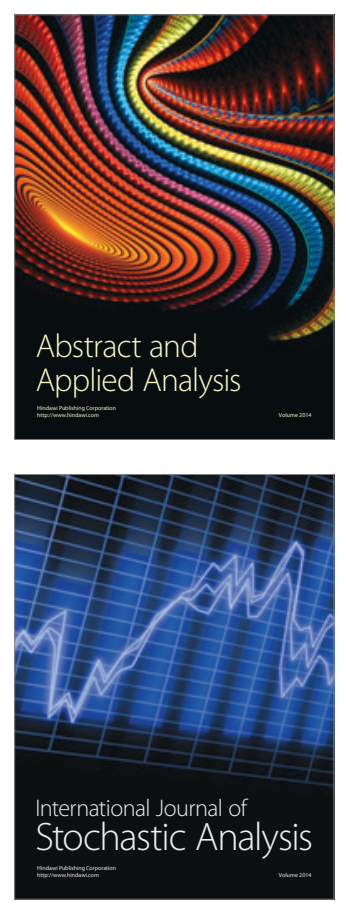

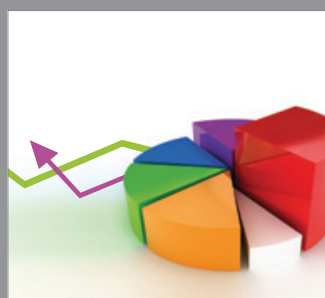

ournal of

Probability and Statistics

Promensencen
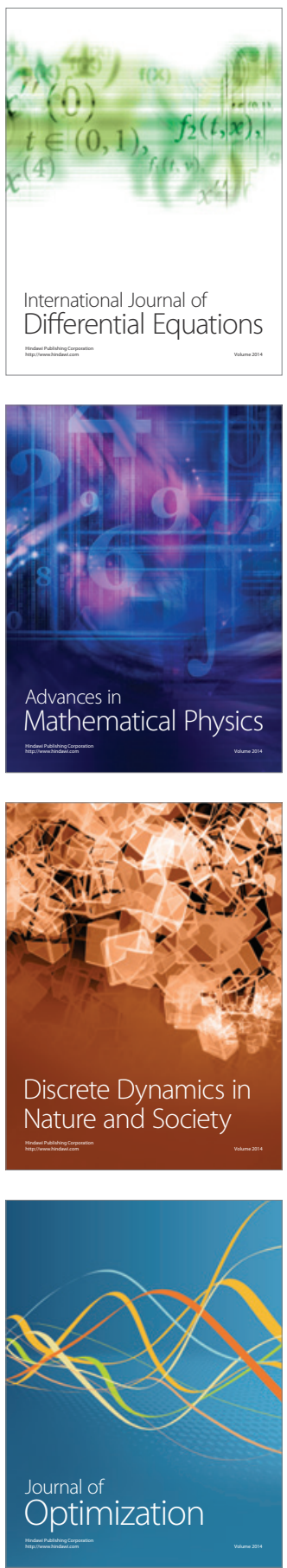